\title{
Identité « propre » ou identité « empruntée » des littératures mineures? Hétérolinguisme dans la traduction littéraire intra- belge
}

\section{Reine Meylaerts \\ KULeuven \\ Introduction}

«Faut-il remercier Dieu d'être Français plutôt que Belge?» se demandait Baudelaire et il jugeait superflu de répondre. Près d'un siècle plus tard, en 1985, son compatriote Pierre Bourdieu se demande «Existe-t-il une littérature belge ? » (1985 : 3-6). Si le père de la théorie des champs daigne bien répondre, c'est pour conclure aux limites d'un champ en mal de structures propres. La production littéraire francophone belge est désignée par Jacques Dubois, collègue belge de Bourdieu, en la même année 1985 comme « une littérature en formation, peu autonome, qui n'a ni trouvé son assise, ni fixé son image », «difficile à définir et à nommer» (1985: 13-20). Dans une perspective comparable - mais à forte orientation française - Pascale Casanova ${ }^{1}$ associe les lettres francophones belges à une "petite littérature »: un espace littéraire démuni, dominé. Déjà en 1938, Gustave Charlier les qualifiait de «littérature seconde: se développant dans une langue qui lui est commune avec la littérature nationale d'un autre pays, en l'occurrence la France » (1938 : 8-9).

Sans être absents, les questionnements existentiels concernant les lettres néerlandophones en Belgique sont moins alarmants et moins fréquents. Très tôt en effet, les Belges néerlandophones ont fait abstraction d'une identité littéraire «nationale », «belge », en associant non pas «langue » et «nation » mais «langue» (le néerlandais) et « ethnie» (les Flamands). L'identité intranationale ainsi créée pouvait s'appuyer sur de solides institutions comme la Koninklijke Academie voor Taal- en Letterkunde en 1886, précédant de plusieurs décennies la naissance d'une institution similaire francophone. N'empêche que la dépendance néerlandophone belge vis-à-vis des structures éditoriales hollandaises reste réelle, notamment dans le domaine des traductions, domaine «délocalisé » par excellence ${ }^{2}$. Bref, le territoire belge semble héberger deux littératures mineures (francophone et néerlandophone) selon la définition de Deleuze et Guattari : une littérature mineure est « celle qu'une minorité fait dans une langue majeure ». Cette langue y est « affectée d'un fort coefficient de déterritorialisation» (1975: 45). Le bilinguisme interne de la nation (français - néerlandais), le partage des langues littéraires nationales

\footnotetext{
${ }^{1}$ Casanova, Pascale (1999), La République mondiale des lettres.

${ }^{2}$ Dirkx, Paul (1995), " Paris and Amsterdam as Translational Go-Betweens. The Evolution of Literary Translation in Belgium after World War II », 13-27.
} 
avec les grands voisins français et néerlandais qui possèdent de surcroît le presque monopole des instances de consécration, les différentes phases de la fédéralisation du pays ${ }^{3}$, sont avancés comme autant d'hypothèques à l'affirmation d'une littérature proprement «belge », forgée selon le modèle français de l'État-nation (une nation, une langue, une littérature).

Or, il va de soi que ces littératures francophone et néerlandophone n'existent pas en isolement l'une à côté de l'autre à l'intérieur du cadre géopolitique belge. Toute culture/littérature se définit en effet en relation avec, voire en opposition à d'autres littératures/cultures. L'analyse des relations littéraires intra-belges peut contribuer par conséquent à appréhender le fonctionnement et la dynamique des littératures en question. Mais comment penser relations entre ces deux littératures mineures ? La question est restée largement en friche à l'intérieur de l'historiographie belge qui, de la sorte, a laissé dans l'ombre des pans entiers de la vie littéraire. Jusqu'à nouvel ordre, rares restent en effet les approches qui délaissent le point de vue bipolaire unidirectionnel et monolingue (France - Belgique francophone ou Pays-Bas - Flandre) pour celui multipolaire, multilingue, inter- et intranational. Rares sont également les études portant sur les pratiques interculturelles, les flux d'importation et d'exportation littéraires, les phénomènes de traduction intra-belge, etc. ${ }^{4} \mathrm{Si} \mathrm{l}^{\prime}$ identité se définit différenciation, si donc les littératures se définissent en opposition à d'autres littératures (au pluriel), il y a lieu de penser cette observation jusque dans ses conséquences intra-nationales et de ne pas limiter les recherches sur les littératures francophone et néerlandophone en Belgique à leurs relations respectives avec la France ou les Pays-Bas. L'ouverture des perspectives que présuppose l'analyse de cette dynamique intra-nationale requiert un modèle de recherche plus souple que celui traditionnel, et très français d'ailleurs, d'un modèle culturel national, territorial, monolingue. Cadre géopolitique multiculturel et multilingue, la Belgique constitue un terrain de choix, un laboratoire pour l'étude comparée des interactions, des relations et des chevauchements entre ses littératures (mineures), leurs fonctions sociales, leurs rapports de force. Bref, dans une situation de multilinguisme une telle étude révélera « une bouillie, une histoire embrouillée, une affaire politique, que les linguistes ne connaissent pas du tout, ne veulent pas connaître » (Deleuze \& Guattari 1975 : 45).

\footnotetext{
${ }^{3}$ Après la Seconde Guerre Mondiale, la Belgique s'est progressivement transformée (1970, 1980,1993) en un pays fédéralisé composé de deux régions monolingues (la Flandre et la Wallonie) et une région bilingue (Bruxelles-Capitale), et de trois communautés (la communauté flamande, francophone et germanophone).

${ }^{4}$ Pour la période de l'entre-deux-guerres, voir par exemple, Meylaerts, Reine (2004a), L'Aventure flamande de la Revue Belge. Langues, littératures et cultures dans l'entre-deux-guerres; Meylaerts, Reine (2004b), «La traduction dans la culture multilingue : À la recherche des sources, des cibles et des territoires », 289-317.
} 
Qui plus est, dans des contextes multilingues où différentes langues, littératures et cultures coexistent, celles-ci maintiennent des relations qui sont par définition hiérarchiques, voire parfois problématiques. En d'autres termes, les deux littératures belges, ces deux littératures mineures que nous venons d'identifier, entretiennent, elles aussi, des relations hiérarchiques. Une des deux littératures mineures en présence fonctionnera comme une littérature majeure dans ses relations avec l'Autre allophone, sans pour cela perdre son statut de littérature mineure par rapport à la littérature majeure homophone. Dans le cas de la Belgique, la littérature francophone belge fera figure de littérature majeure vis-à-vis de la littérature néerlandophone belge tout en subissant la domination de la littérature française. Les relations hiérarchiques entre différentes littératures et cultures à l'intérieur d'un espace géopolitique multilingue sont entre autres créées par des structures institutionnelles et discursives. Quelle est la langue ou quelles sont les langues des institutions «nationales » (administration, enseignement, justice, armée, etc.) d'une part et quelles sont les dichotomies positives et négatives qui structurent les pratiques discursives vis-à-vis de l'Autre, sont dès lors des questions-clé pour étudier les hiérarchies culturelles et pour comprendre la dynamique des relations interculturelles dans un contexte multilingue donné. Voyons comment ces paramètres se présentent dans le cas de la Belgique.

\section{Un État-nation et ses langues}

Il y a peu de sociétés dans lesquelles les questions de langue et d'identité sont aussi complexes, changeantes et problématiques qu'en Belgique. Ceci peut paraître étonnant dans la mesure où, en comparaison avec beaucoup de pays africains où des centaines de langues se partagent un seul territoire, ce très petit État occidental n'a «que» trois langues officielles aujourd'hui : le français, l'allemand ${ }^{5}$ et le néerlandais. Bien sûr, des relations linguistiques problématiques sont moins liées au nombre de langues impliquées qu'à la perception des valeurs et des oppositions linguistiques et donc à des idéologies de langue.

Concrétisons. À sa création, en 1830, le choix du français comme langue de l'administration, de la justice, de l'enseignement et de l'armée pour la jeune nation belge était hors discussion. Le français s'était en effet affirmé comme langue de culture, scientifique et diplomatique de l'Europe: son rayonnement allait être garant d'un avenir en rose pour le pays nouveau-né.

\footnotetext{
${ }^{5}$ L'allemand est parlé dans les soi-disant cantons de l'Est où habite une petite minorité germanophone annexée en 1920 en exécution du traité de Versailles. Actuellement, le statut de l'allemand est légèrement différent de celui du français et du néerlandais. Puisque les Germanophones ne se sont jamais mêlés des luttes linguistiques, nous ne les prenons pas en considération dans cette étude.
} 
Pourtant, ce monolinguisme institutionnel contrastait avec le multilinguisme sur le terrain, où l'usage des langues était déterminé tant par des facteurs géographiques (le néerlandais au nord et le français au sud) que sociaux. Les élites politiques, économiques et culturelles au nord et au sud, parlaient le français standard. Les classes populaires, avec un pourcentage important d'illettrés pendant le $19^{\text {e }}$ siècle ${ }^{6}$, parlaient des dialectes: des dialectes néerlandais (souvent dédaigneusement appelés 'flamands') au nord et des dialectes wallons au sud. Les classes moyennes, bénéficiant de l'industrialisation progressive, avaient un accès croissant à l'enseignement. Ayant suivi l'enseignement français, elles devenaient progressivement bilingues selon un principe diglossique. Elles utilisaient le français standard dans la sphère publique, à l'école, dans leurs contacts avec les classes supérieures et le dialecte dans les situations informelles, en famille, pour la communication avec les classes inférieures. En particulier au nord, cette diglossie impliquait des relations hiérarchiques, voire oppositionnelles entre le français et le néerlandais, ce dernier souffrant à travers le $19^{\text {e }}$ et jusqu'au début du $20^{\mathrm{e}}$ siècle de son image dialectale, inférieure.

Bref, les langues étaient liées à des phénomènes de pouvoir et de prestige social, culturel et politique et des mécanismes institutionnels contribuaient à contrôler qui «était dedans » et qui «était dehors ». Les pratiques langagières étaient effectivement indexées, le néerlandais étant quasiment exclu de la politique, de l'administration, de l'enseignement, et de l'appareil judiciaire. Cet état des choses avait évidemment des implications fondamentales pour $l^{\prime}$ habitus ${ }^{7}$ linguistique des Belges. À travers ces structures institutionnelles monolingues ainsi que par le biais de pratiques discursives qui confirmaient constamment la supériorité du français et l'infériorité du néerlandais, l'adulte moyen du $19^{\mathrm{e}}$ et du début $\mathrm{du} 20^{\mathrm{e}}$ siècle avait intériorisé, de façon variable et variée, une telle hiérarchie linguistique. Lisons un exemple type des termes dans lesquels cette supériorité se manifestait au début des années 1920 :

Dès le début du $\mathrm{XI}^{\mathrm{e}}$ siècle, le français s'introduisit en Flandre avec les idées nouvelles et toute la civilisation de l'Europe médiévale, sans violence, par la force même des choses.

\footnotetext{
${ }^{6}$ Ce n'est qu'en 1914 que la Belgique introduisit la scolarité obligatoire jusqu'à l'âge de 12 ans.

${ }^{7}$ Le concept de « habitus » est développé par Pierre Bourdieu. Il désigne le système intériorisé de structures sociales sous forme de dispositions durables. Sous l'influence de sa position sociale et de son passé individuel et collectif, tout agent culturel développe (et continue à développer) une certaine représentation du monde et de sa position dans le monde, une 'identité sociale'. Voir entre autres Bourdieu, Pierre (1972), Esquisse d'une théorie de la pratique.
} 
Que le français ait prédominé sur le flamand, notre bon sens nous le dit, et l'histoire nous l'apprend. [...]

[...] entre connaître la langue flamande et recevoir toute son instruction dans cette langue, il y a un abîme. Empêcher les jeunes flamands [sic] de recevoir leur instruction en langue française, c'est leur enlever le plus beau patrimoine intellectuel, qu'ils puissent posséder. En effet, la langue française, est une langue mondiale, l'organe d'une puissante civilisation, un instrument de tout premier ordre pour la diffusion des idées, et pour l'élargissement du cercle mental où se meut le peuple flamand. ${ }^{8}$

Le français était perçu comme langue de distinction socioculturelle et de mobilité sociale; la connaissance du français déterminait l'accès à l'université et aux professions prestigieuses. En outre, les productions littéraires les plus légitimes étaient écrites en langue française. Plus en particulier, la définition à succès d'une littérature «nationale » «belge » se réduisait à des œuvres en langue française mais puisant dans un imaginaire flamand poétisé que fournissait le riche passé culturel des Flandres ${ }^{9}$. «Belge » égalait donc «langue française» + «inspiration flamande ». Cette formule, résumée par J.-M. Klinkenberg sous l'expression de «mythe nordique » faisait abstraction de la dimension bilingue du pays en faveur d'une vision unilingue francophone (1989: 33-50). Elle fonctionnait comme la définition identitaire officielle d'une littérature «belge » jusque dans la première moitié du $20^{\mathrm{e}}$ siècle, tout en présupposant un rapport hiérarchique inégal entre les langues, les littératures et les cultures en Belgique au profit de la seule composante francophone. Peu à peu, cependant, des voix discordantes commençaient à s'élever.

Surtout les membres des classes moyennes néerlandophones considéraient cette domination institutionnelle et symbolique du français comme une injustice puisqu'elle compliquait leur accès aux professions prestigieuses et donc leur ascension sociale. Le monolinguisme institutionnel renforçait en effet les structures sociales d'inégalité dans une société multilingue. Par conséquent, le Mouvement flamand exigeait - timidement d'abord, mais avec plus d'insistance dès le début du $20^{\mathrm{e}}$ siècle - pour le néerlandais les attributs et les domaines du pouvoir dont il avait été exclu

\footnotetext{
${ }^{8}$ Gandavus, André (sans date), La Question flamande jugée par un Flamand, 4-5. Pour d'autres exemples de pratiques discursives (littéraires et autres), le lecteur se référera à Meylaerts (2004a), Aventure, et Meylaerts (2007) « "La Belgique vivra-t-elle?" Language and Translation Ideological Debates in Belgium (1919-1940) », 297-319.

${ }^{9}$ Le concept « Flandre » n'y réfère pas à un territoire défini de façon univoque qui coïnciderait avec la Région flamande actuelle, mais réunit un amalgame d'éléments mythiques (mysticisme, brumes nordiques, pittoresque, kermesses populaires, etc.).
} 
auparavant. Le néerlandais devait être accepté comme langue officielle de l'administration, de la justice et de l'enseignement au nord du pays. Bien sûr, puisque tout changement de politique linguistique institutionnelle est facilement interprété comme un danger potentiel pour la cohérence et même la survie de la nation, ceci était un procès long et difficile. Les lois les plus importantes créant des institutions néerlandophones monolingues basées sur le principe du territorialisme (le néerlandais au nord et le français au sud), étaient votées dans les années 1930, un siècle après l'indépendance.

Parallèlement, la littérature néerlandophone s'émancipait progressivement de son statut inférieur. En 1907, August Vermeylen, Flamand bilingue, docteur en histoire, écrivain et futur premier recteur de l'Université néerlandophone de Gand (1930), faisait une conférence à l'occasion de l'exposition universelle à Liège. Devant son auditoire francophone (belge et étranger), Vermeylen évoquait l'ignorance, voire l'inexistence dont avaient souffert les lettres néerlandophones jusqu'à récemment :

Il ne me sera probablement pas possible de vous faire sentir, de façon assez claire et assez vive, ce qu'a été, dans ses grandes lignes, la littérature flamande. Mais je pourrai toujours vous convaincre de son existence, et c'est déjà beaucoup. (1907: 5)

Quelque vingt ans plus tard, certains semblaient se réjouir d'une évolution positive à l'intérieur de la Belgique : «Le temps n'est plus » écrivit le Flamand bilingue André de Ridder dans son introduction à La littérature flamande contemporaine, "où, sous prétexte de glorifier "l'âme belge », on commençait par en négliger et en supprimer la bonne moitié » (1923 : 38). Bref, les lettres néerlandophones commençaient à exiger leur place dans le panthéon littéraire national dès le début du $20^{\mathrm{e}}$ siècle.

Les élites francophones percevaient ces évolutions sociopolitiques et littéraires comme des menaces pour leur hégémonie politique et culturelle (voir Meylaerts, Aventure). En d'autres mots, les premières décennies du $20^{\mathrm{e}}$ siècle constituaient une période de crise identitaire importante pendant laquelle les anciennes hiérarchies politiques, linguistiques et littéraires perdaient de leur évidence apparente et étaient questionnées - sans pour autant être radicalement bouleversées.

\section{Lorsqu'une littérature majeure traduit une littérature mineure}

C'est dans ce contexte, et plus précisément pendant les années 1920-1930, que le nombre de traductions littéraires du néerlandais en français en Belgique 
connaissait une montée considérable. Cet intérêt de la littérature majeure francophone, elle-même mineure vis-à-vis le grand voisin français, pour la littérature mineure néerlandophone se manifestait tant par des traductions en volume que par des traductions dans des revues. D'un point de vue quantitatif, toutes langues sources confondues, environ $50 \%$ des volumes traduits en français et publiés en Belgique étaient des traductions littéraires. Tandis qu'avant 1918 et après 1945 l'allemand était la première langue cible pour les traductions de littérature néerlandophone, le français était la principale langue cible pendant l'entre-deux-guerres. Plus de $75 \%$ de ces traductions du néerlandais en français étaient publiées par une maison belge et fonctionnaient donc uniquement en Belgique, pas en France. Comme déjà examiné, le marché littéraire français était en effet structurellement et symboliquement dominant par rapport au marché belge francophone. Par conséquent, des livres publiés en Belgique avaient un capital symbolique trop bas pour pénétrer en France. Bref, le quasi monopole des éditeurs belges francophones dans le domaine des traductions belges néerlandophones contrastait violemment avec la véritable colonisation $\mathrm{du}$ marché belge francophone par les maisons françaises dans d'autres domaines. En somme, l'entre-deux-guerres était une période de contacts traductionnels privilégiés entre les cultures francophone et néerlandophone à l'intérieur du cadre géopolitique belge. Les observations suivantes s'appuient sur l'analyse d'un échantillon représentatif de ces traductions. Il regroupe une trentaine de nouvelles ou de romans traduits en volume ou dans les revues les plus représentatives de l'époque ${ }^{10}$.

À en croire d'innombrables pratiques discursives francophones concernant ces traductions du néerlandais en français, le public cible de ces traductions était les élites monolingues francophones belges qui se sentaient menacées par les exigences d'émancipation sociopolitique et culturelle des Flamands ${ }^{11}$. Ils s'intéressaient aux traductions pour des motifs patriotiques. Les comptes rendus que leur presse consacrait à ces traductions y insistaient souvent explicitement :

Quel livre écrit en français m'eut permis de me faire une idée de la littérature flamande contemporaine?... [...] Ce livre nécessaire vient enfin de paraître. C'est La Littérature flamande contemporaine de M.

\footnotetext{
${ }^{10}$ Entre 1918 et 1919, au total quelque 90 volumes néerlandophones paraissaient en traduction française. S'y ajoutaient d'innombrables publications en revue, dont la plus importante était la Revue Belge. Pour des données bibliographiques, le lecteur se référera à Arents, Prosper (1931), De Vlaamsche Schrijvers in vertaling. 1830-1931. Proeve van Bibliographie; Pépin, JeanPierre (1972), Essai de bibliographie des traductions françaises des cuvres de la littérature néerlandaise depuis 50 ans (1918-1968).

${ }^{11}$ Pour des exemples, voir entre autres Meylaerts (2004a), Aventure, 148-158.
} 
André De Ridder. Je recommande cet ouvrage à tous les belges [sic] qui n'entendent point le flamand. J'espère, pour eux, qu'ils ressentent, comme moi, un grand désir de connaître cette littérature. J'espère qu'ils ne se détournent pas des flamands [sic] et de l'âme flamande à cause des luttes linguistiques qui encombrent la politique intérieure de notre pays. À cause de ces luttes mêmes il importe, au contraire, de bien connaître les flamands [sic] et leur âme. Il faut savoir non pas seulement ce qu'ils veulent, mais aussi ce qu'ils valent. Il faut comprendre surtout que nous sommes solidaires. Ils sont comme nous, les intermédiaires naturels entre la civilisation germanique et la civilisation franco-latine. ${ }^{12}$

Cette initiative revêt un caractère d'intérêt national. [...] Les traductions du flamand en français et du français en flamand devraient être plus nombreuses pour que chacun puisse comprendre d'une façon positive cet esprit national que les Picard et les Kurth nous ont révélé. Pour continuer le passé, notre pays a besoin de ses deux littératures; ce sont les deux ailes pour un essor harmonique et durable. ${ }^{13}$

Les traductions étaient accueillies comme un service à la nation et à sa littérature nationale: elles devaient aider à surmonter les tensions linguistiques et sociopolitiques par l'union des deux cultures. Raisonnement précieux. Dans un contexte de fortes tensions, voire de luttes linguistiques, la traduction dans la langue majeure conditionnait le contact avec la langue et la littérature mineures; elle fonctionnait donc comme une confirmation de la position prestigieuse du français comme unique langue (littéraire) nationale. Face à une situation de relative mise en péril de la définition légitime de l'identité nationale (littéraire et autre), il s'agissait de conserver au terme «belge » son acception privilégiée : « de langue française ${ }^{14}$.

Les caractéristiques génériques des textes traduits s'avéraient remarquablement uniformes : c'était la nouvelle régionaliste qui monopolisait les contacts traductionnels. Elle exaltait la vie campagnarde traditionnelle d'une Flandre paisible et pittoresque et dépeignait des personnages populaires, pauvres, souvent drôles. Au sein de la littérature néerlandophone, les récits régionalistes formaient à ce moment le genre à succès des auteurs néerlandophones les plus en vogue. C'était une littérature qui mettait en scène les classes populaires et moyennes néerlandophones auxquelles elle

\footnotetext{
${ }^{12}$ Gilkin, Iwan (1924), « Chronique littéraire » Revue Belge, 492.

${ }^{13}$ Sosset, Léon-Louis (1931), «LES LIVRES », La revue nationale, 973-974.

${ }^{14}$ Ceci explique pourquoi certains néerlandophones considéraient les traductions comme une trahison de la cause flamande et s'opposaient à ce genre de contacts interculturels.
} 
s'adressait. En ce sens, elle pouvait jouer un rôle émancipatoire pour la Flandre. Au cours des années trente, le genre allait en effet être contesté par une nouvelle garde qui prônait une littérature plus "moderne ", davantage affiliée aux mouvements modernistes étrangers. La traduction en français, quant à elle, signifiait un transfert à la fois linguistique et socioculturel. Aux yeux du nouveau public cible, les élites francophones, l'intrigue confirmait les clichés d'un imaginaire flamand poétisé : elle reflétait un monde réconfortant où «flamand » était synonyme de fêtes populaires bruyantes, de kermesses, mais également de brumes nordiques, de recueillement religieux, de pauvreté, de grisaille, bref, de « classe populaire ».

L'écurie de Monsieur Jules [l'âne] n'en était pas une. Il gîtait en fait dans la maison même du marchand de moules. Lui à gauche; à droite les mollusques et un peu de fourrage; au-dessus le colporteur et sa femme. Une autre petite pièce devait exister là-haut, car, le matin, une horde innombrable de mioches, garçonnets et fillettes, dégringolait l'échelle. Plusieurs des plus petits criaient papa, un autre bon-papa. ${ }^{15}$

Une expression bien définie résumait cette fonction idéale dans les yeux des élites francophones: "simplement flamand ». L'expression était omniprésente dans le discours littéraire francophone (comptes rendus de traductions, articles, etc.), ainsi que dans les archives personnelles des traducteurs, éditeurs, etc. Lisons comment le directeur de la Revue Belge, la revue qui publiait le plus grand nombre de traductions flamandes dans les années 1920-1930, explicitait sa politique de traduction :

Je recherche autant que possible, dans les traductions flamandes, des œuvres qui ont un caractère simplement flamand, telles que celles que tu nous as d'ailleurs données jusqu'à présent $(. ..){ }^{16}$

Cette caractéristique conditionnait la frontière entre la traduction et la non traduction: les genres plus modernistes, liés à des mouvements internationaux n'étaient quasiment jamais traduits. Symbolisant une Flandre plus moderne, l'émancipation socioculturelle dangereuse de la culture mineure, ils n'étaient pas acceptés par les élites francophones.

\section{Les langues de la traduction}

Comme nous l'avons $\mathrm{vu}$, pendant l'entre-deux-guerres belge les langues fonctionnaient comme emblème d'un groupe social. Ces relations

\footnotetext{
${ }^{15}$ Van Cauwelaert, August (1935), «L'heure du baudet », Revue Belge, 59.

${ }^{16}$ Goemaere, Pierre (directeur de la Revue Belge) (1932), Lettre à Roger Kervyn de Marcke ten Driessche, traducteur. Pour d'autres exemples, voir Meylaerts (2004a), Aventure.
} 
problématiques, voire conflictuelles entre les langues et littératures majeure et mineure influençaient également les modalités et fonctions traductionnelles (et vice versa). Par conséquent, parmi les questions-clé pour comprendre la dynamique des relations interculturelles dans un espace géopolitique multiculturel comme la Belgique de l'entre-deux-guerres, une analyse des conditions de possibilité, des modalités et fonctions de l'hétérolinguisme littéraire $^{17}$ dans les traductions françaises de fiction néerlandophone se révèle particulièrement intéressante. Jusqu'à quel point une traduction pouvait/devait-elle afficher un caractère «flamand» dans sa réalisation textuelle? Comment cette question était-elle connectée aux modalités et fonctions de l'hétérolinguisme dans les textes sources néerlandophones?

Bon nombre d'auteurs régionalistes néerlandophones étaient appréciés à l'époque par les lecteurs néerlandophones pour un certain type d'hétérolinguisme littéraire: une alternance codique entre le néerlandais standard et les dialectes ou les variantes régionales et, parfois, même le français. Cette alternance codique apparaissait surtout dans les dialogues, en fonction de l'origine sociale des personnages. Les auteurs flamands utilisaient des dialectes et/ou des variantes régionales pour les classes populaires, le néerlandais standard ou le français pour les classes moyennes et le français pour les élites. Le recours au français était également réservé à certaines situations plus ou moins officielles : à l'école, devant la cour de justice, dans les grandes villes, etc. L'usage de dialectes dans les dialogues répondait à des motifs mimétiques (véracité) et identitaires: les classes populaires néerlandophones avaient droit à l'existence dans une littérature spécialement produite pour elles et pour l'émancipation littéraire et culturelle flamande.

Une des caractéristiques les plus frappantes que se partagent les traductions françaises des années 1920-1930 est l'absence totale de dialectes et de variantes régionales. Les dialectes néerlandais n'étaient jamais traduits par des dialectes wallons. Puisque les langues cristallisaient des positions sociopolitiques, l'usage du wallon aurait été perçu par les élites francophones comme une atteinte au statut $\mathrm{du}$ français comme l'unique langue nationale littéraire légitime, voire comme une option en faveur des exigences séparatistes du mouvement wallon ${ }^{18}$. En d'autres termes, les dialectes wallons auraient été incompatibles avec la fonction patriotique des traductions: la

\footnotetext{
${ }^{17}$ Par hétérolinguisme ou pluralité langagière nous entendons la présence dans le texte d'éléments étrangers ou de variétés régionales, historiques, sociales..., considérés non pas d'un point de vue anecdotique ou normatif, mais d'un point de vue fonctionnel, institutionnel. Voir également : Grutman, Rainier (1997), Des langues qui résonnent: l'hétérolingü̈sme au XIX siècle québécois ; Meylaerts, Reine (2006), " Heterolingualism in/and Translation. How legitimate are the Other and his/her Language? », Target, 1-15.

${ }^{18}$ Pour de plus amples détails concernant le statut du wallon et son inacceptabilité dans les traductions françaises pendant l'entre-deux-guerres belge, voir Meylaerts (2004a), Aventure.
} 
construction-continuation d'une littérature nationale monolingue francophone belge et la glorification de l'État-nation monolingue francophone. En outre, les auteurs flamands néerlandophones s'opposaient à l'utilisation de dialectes dans les traductions parce que cela aurait compromis une éventuelle percée de leur œuvre en France. Espoir vain dans la plupart des cas (cf. plus haut). Pour ces deux raisons, un aspect fondamental d'hétérolinguisme dans les textes originaux néerlandophones disparaissait dans les traductions françaises.

Est-ce que cela signifie que toute représentation linguistique du caractère «flamand » dans les traductions françaises faisait défaut? Pas du tout. Un ensemble d'options alternatives symbolisait le caractère flamand et les hiérarchies sociolinguistiques en vigueur sans violer les sensibilités sociolinguistiques de la culture majeure. Une première stratégie concernait le maintien des toponymes et patronymes néerlandophones, parfois même les diminutifs ou les sobriquets patoisants des personnages:

Derrière eux venait Guustje, le frère du défunt, accompagné de Léo, d'Angelus, de Célestien, de Deefiel et d'autres hommes habitant le hameau. Leurs femmes: Meelnie, Siednie, Eemlie, Falderie, Emérance et Kathelynsten. ${ }^{19}$

Cette option identifiait clairement le cadre géographique et les personnages comme «flamands» dans leur réalisation hétérolingue. L'histoire se déroulait à la campagne flamande, dans de petits villages paysans avec des protagonistes appartenant aux classes populaires néerlandophones. Pour les lecteurs des élites francophones, les toponymes et patronymes flamands évoquaient un monde familier de villages dans lesquels ils vivaient parfois eux-mêmes (mais en tant que châtelain local, par exemple) avec des hiérarchies sociolinguistiques rassurantes et avec des personnages ayant les noms de leurs domestiques. Ceci n'était pas la Flandre dangereuse avec ses exigences d'émancipation sociolinguistique. Ce type d'éléments hétérolingues flamands dans les traductions françaises n'affectait par conséquent pas le statut du français comme langue nationale littéraire prestigieuse. En outre, les noms propres néerlandophones étaient courants dans les récits originaux français et faisaient dès lors partie du répertoire littéraire de l'époque.

Une autre stratégie, encore plus fondamentale, était la traduction des dialectes flamands par des sociolectes français. Dans sa version minimale, la traduction alternait entre le français standard pour la narration et le français

\footnotetext{
${ }^{19}$ Buysse, Cyriel (1931), « Le repas des funérailles en Flandre. Traduit du flamand par Pierre
} Maes », Revue Belge, 41. 
familier, populaire pour les dialogues. La différenciation sociale et régionale des dialectes flamands était donc réduite à la différenciation sociale des sociolectes. Les personnages des traductions n'étaient pas des Wallons appartenant aux classes populaires patoisantes du sud (cf. plus haut) mais s'identifiaient sans équivoque comme des Flamands néerlandophones appartenant aux classes populaires.

«Ah, ça oui, m'sieur », répondit Poover $(\ldots)^{20}$.

«Ce n'est pas d'refus », répondit Poover du même ton rauque ${ }^{21}$.

Il brandit, menaçant, son fouet :

- Faut qu'il ouvre, sinon je renverse la maison de l'équarisseur, l'équarisseur lui-même, sa servante et tout le tremblement ${ }^{22}$.

Cette option présentait deux avantages. D'abord elle renforçait la synonymie entre «Flamand» et «populaire» que la culture dominante désirait soutenir : l'association de ce style particulier à un ton "populaire » était explicitement appréciée par les lecteurs francophones. Elle confirmait en d'autres termes les élites francophones dans leur perception des hiérarchies sociolinguistiques et socioculturelles. Ensuite, l'usage de sociolectes permettait d'éviter non seulement les dialectes mais également d'autres types d'interférences traductionnelles comme les flandricismes, les belgicismes et autres. Dans la limitation consciente du vocabulaire à des mots présents dans n'importe quel dictionnaire du français standard, cette option laissait à son tour intacte la langue française, symbole de la nation et de sa littérature la plus légitime.

À cause de son succès dans la culture majeure, certains traducteurs développaient une version plus élaborée de ce type d'hétérolinguisme. Ils ne remplaçaient pas uniquement les dialectes des dialogues mais également le néerlandais standard des dialogues et du récit par le français populaire et familier (voir les parties soulignées dans l'extrait ci-dessous). Le néerlandais standard, symbole de l'émancipation des classes flamandes moyennes était traduit dans une version plus populaire, hétérolingue. Une fois de plus, « Flamand » était réduit à « simplement flamand » en accord avec les attentes de la littérature et culture majeures.

Par exemple, le soir, quand on avait porté au berceau dans la chambre à coucher ce petit trésor d'enfant, il suffisait que papa dit à maman, d'un ton enjoué : "Voilà chérie, nous allons passer ensemble une

\footnotetext{
${ }^{20}$ Buysse, Cyriel (1925), « Le Solitaire. Traduit du flamand par André de Ridder », Revue Belge, 62.

${ }^{21}$ Ibid.

${ }^{22}$ Van Cauwelaert, August (1935), op cit., 65.
} 


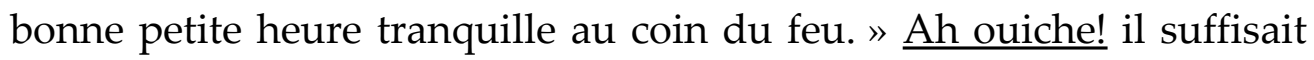
de ça pour que, de la pièce contiguë, s'élevât une protestation de Kiki, véhémente à faire croire qu'il s'était enfoncé une épingle dans le cuir. La « petite heure tranquille » était au diable, comme de juste: maman volait chaque fois vers le marmouset déchaîné et, lui, trouvait toujours le joint pour la retenir le plus longtemps possible ${ }^{23}$.

Il est finalement intéressant de jeter un coup d'œil sur la traduction de dialogues français présents dans certaines œuvres flamandes néerlandophones. Dans l'original, les passages en français étaient des instances de contact entre les langues et cultures majeure et mineure. Ils étaient utilisés pour les prises de parole des classes supérieures ou moyennes. Les traductions en français rendaient, par définition, ces contacts invisibles. Or, par l'insertion d'une note infrapaginale (« en français dans l'original »), les traductions françaises attiraient l'attention sur l'hétérolinguisme du texte source, symbole du désir d'ascension sociale des classes moyennes et de leur intériorisation des hiérarchies sociolinguistiques existantes.

Dans l'exemple suivant, un père flamand chante des chansons estudiantines et de troupiers en français pour son fils, confirmant ainsi le français comme langue de l'université et de l'armée. Grâce à la note du traducteur, cette information, rassurante aux yeux des lecteurs francophones, passe également dans la traduction en français.

De sorte que, à la vive indignation de sa maman, les chansons enfantines de Kiki furent des refrains d'étudiants ou de troupiers. [...]

Elle avait une jambe en bois

Et pour que ça ne se vit pas

Elle avait mis en dessous

Une rondelle en caoutchouc. $\left(^{*}\right)$

Jusqu'à ce que maman intervînt, [...]

-C'est-il des chansons pour un enfant, voyons?

-Bah! C'est pour ça que je chante en français. Il ne comprend pas !

$\left.{ }^{*}\right)$ En français dans le texte ${ }^{24}$.

\section{Conclusion}

Lorsque la traduction sert à la glorification d'une nation officiellement monolingue dans un contexte multilingue de tensions sociolinguistiques, la langue de la traduction est chargée d'une importance symbolique du plus

\footnotetext{
${ }^{23}$ Claes, Ernest (1929), « Kiki. Traduit du flamand par Roger Kervyn de Marcke ten Driessche », Revue Belge, 519-520.

${ }^{24}$ Ibid., 529.
} 
haut degré : elle accentue les lignes de partage idéologiques, socioculturelles de la société et forme une prise de position concernant l'identité culturelle. Une étude des différentes conditions de possibilité, formes et fonctions de l'hétérolinguisme littéraire dans la fiction traduite d'une langue mineure en une langue majeure peut donc révéler des aspects essentiels de la construction et de la dynamique de l'identité culturelle dans une société multiculturelle. Dans la Belgique francophone de l'entre-deux-guerres, la langue de la traduction évite de se présenter comme un instrument d'émancipation sociolinguistique. L'idéologie de la pureté est plus forte que la recherche de la vraisemblance linguistique. Les effets de traduction sont incompatibles avec l'image de la langue majeure comme la langue nationale littéraire et culturelle par excellence. La langue cible majeure est affirmée dans sa supériorité, non affectée par le contact traductionnel avec la langue mineure.

Plus en général, dans un environnement géopolitique multilingue où l'homogénéité territoriale en termes de population, de langue et de normes socioculturelles est relative et où de nombreux messages transgressent les frontières linguistiques et sociales, il s'avère particulièrement pertinent d'investiguer comment et pourquoi l'hétérolinguisme est présent ou absent dans la prose littéraire traduite. Jusqu'à quel point une traduction peut-elle (ou devrait-elle) être hétérolingue dans un contexte particulier ? Quelles sont les modalités et les fonctions identitaires de l'hétérolinguisme littéraire dans la traduction littéraire ? Et comment sont-elles, oui ou non, liées aux modalités et aux fonctions identitaires de l'hétérolinguisme littéraire dans des textes « originaux» appartenant aux cultures source et cible? Se trouvent-elles en rapport avec le discours sur «l'Autre », avec la doxa sur la traduction dans des contextes particuliers, etc.? Jusqu'ici, ces questions n'ont pas reçu l'attention qu'elles méritaient. Implicitement ou explicitement, la traduction est toujours définie comme la transposition complète $\mathrm{d}^{\prime} u n e$ langue source (monolingue) en une langue cible (monolingue) pour un public cible monolingue. Cette définition fait abstraction d'au moins deux facteurs significatifs. D'abord le fait que, par définition, les discours ne sont jamais entièrement monolingues et que, au lieu de refléter simplement la polyglossie sociale, les discours «originaux» et «traduits» sélectionnent certains éléments (hétérolingues) pour représenter symboliquement cette polyglossie. Ensuite, le fait que le monolinguisme des auteurs, des critiques et des publics dans les cultures source et cible, peut être moins absolu qu'on ne le supposerait, surtout dans des contextes multilingues ou soi-disant « globaux ».

Puisque la traduction est un processus interculturel entre des cultures à relations de pouvoir inégales, son degré d'hétérolinguisme peut assumer des fonctions symboliques importantes. Suite à la surdétermination contextuelle 
de contextes minoritaires, les textes traduits peuvent devenir un espace où des conflits de langue et d'identité sont textualisés (Millán-Varela 2004 : 52). $\mathrm{Vu}$ que la langue «nationale» «standard» est le pôle langagier le plus traduisible, la réinsertion de l'opacité entre les langues devient un moyen de résister à la domination et de créer de l'autonomie. Par conséquent, des études descriptives fonctionnelles sur l'hétérolinguisme en/et traduction sont en mesure de corriger le caractère artificiellement monolingue des modèles traductologiques. Elles permettront de mieux comprendre la construction des identités littéraires et la dynamique culturelle dans des contextes multilingues et multiculturels passés et présents. 


\section{BIBLIOGRAPHIE}

ARENTS, Prosper (1931), De Vlaamsche Schrijvers in vertaling. 1830-1931. Proeve van Bibliographie, Bruxelles, Standaard Boekhandel.

BOURDIEU, Pierre (1972), Esquisse d'une théorie de la pratique, Genève, Droz. BOURDIEU, Pierre (1985), «Existe-t-il une littérature belge? Limites d'un champ et frontières politiques ", Études de Lettres. Revue de la Faculté des lettres de l'Université de Lausanne 4, 3-6.

BUYSSE, Cyriel (1925), «Le Solitaire. Traduit du flamand par André de Ridder », Revue Belge ( ${ }^{\text {er }}$ janvier), 57-69.

BUYSSE, Cyriel (1931), «Le repas des funérailles en Flandre. Traduit du flamand par Pierre Maes », Revue Belge ( $1^{\mathrm{er}}$ juillet), 41-46.

CASANOVA, Pascale (1999), La République mondiale des lettres, Paris, Seuil.

CHARLIER, Gustave (1938), Les lettres françaises de Belgique: esquisse historique, Bruxelles, La Renaissance du Livre.

CLAES, Ernest (1929), "Kiki. Traduit du flamand par Roger Kervyn de Marcke ten Driessche », Revue Belge (15 juin), 519-536.

DE RIDDER, André (1923), La littérature flamande contemporaine (1890-1923), Anvers/Paris, Opdebeek/Champion.

DELEUZE, Gilles et Félix GUATTARI (1975), Kafka. Pour une littérature mineure, Coll. Critique, Paris, Minuit.

DIRKX, Paul (1995), « Paris and Amsterdam as Translational Go-Betweens. The Evolution of Literary Translation in Belgium after World War II », dans Peter Jansen (dir.), Translation and the Manipulation of Discourse. Selected Papers of the CERA Research Seminars in Translation Studies 1992-1993, Leuven, The CETRA Chair for Translation, Communication and Cultures, 13-27.

DUBOIS, Jacques (1985), «Jeu de forces et contradictions dans le champ littéraire de la Belgique contemporaine », dans Lise Gauvin et Jean-Marie Klinkenberg (dirs.), Trajectoires. Littérature et institutions au Québec et en Belgique francophone, Bruxelles, Labor, 13-20.

GANDAVUS, André (s.d.), La Question flamande jugée par un Flamand, Ghent, Imprimerie Van Doosselaere (n.d. [1918/19]).

GILKIN, Iwan (1924), "Chronique littéraire », Revue Belge (1 ${ }^{\mathrm{er}}$ mars), 491-500.

GOEMAERE, Pierre (1932), Lettre à Roger Kervyn de Marcke ten Driessche, traducteur en date du 17 septembre 1932. Lettre conservée aux Archives et Musée de la Littérature, Centre de recherche et de documentation littéraires et théâtrales de la Communauté française de Belgique, ML4331/475.

GRUTMAN, Rainier (1997), Des langues qui résonnent : l'hétérolinguïsme au XIX siècle québécois, Québec, Fides.

KLINKENBERG, Jean-Marie (1981), «La production littéraire en Belgique francophone. Esquisse d'une socio-historique », Littérature 44, 33-50.

MEYLAERTS, Reine (2004a), L'Aventure flamande de la Revue Belge. Langues, littératures et cultures dans l'entre-deux-guerres, Bruxelles, P.I.E.-Peter LangAML. 
MEYLAERTS, Reine (2004b), « La traduction dans la culture multilingue : À la recherche des sources, des cibles et des territoires », Target 16.2, 289-317.

MEYLAERTS, Reine (2006), «Heterolingualism in/and Translation. How legitimate are the Other and his/her Language? », Target 18.1, 1-15.

MEYLAERTS, Reine (2007), " “La Belgique vivra-t-elle?" Language and Translation Ideological Debates in Belgium (1919-1940) », The Translator 13.2, 297-319.

MILLÁN-VARELA, Carmen (2004), «Hearing Voices: James Joyce, narrative voice and minority translation », Language and Literature 13.1, 37-54.

PÉPIN, Jean-Pierre (1972), Essai de bibliographie des traductions françaises des cuvres de la littérature néerlandaise depuis 50 ans (1918-1968), Bruxelles, Commission belge de Bibliographie.

SOSSET, Léon-Louis (1932), «LES LIVRES. », La Revue Nationale (15 août-15 septembre), 973-974.

VAN CAUWELAERT, August (1935), «L'heure du baudet. Traduit du flamand par Roger Kervyn de Marcke ten Driessche », Revue Belge (1 ${ }^{\mathrm{er}}$ juillet), 57-70.

VERMEYLEN, August (1907), Les lettres néerlandaises en Belgique depuis 1830. Conférence faite à l'exposition universelle de Liège en 1905, Bruxelles, Lamertin. 